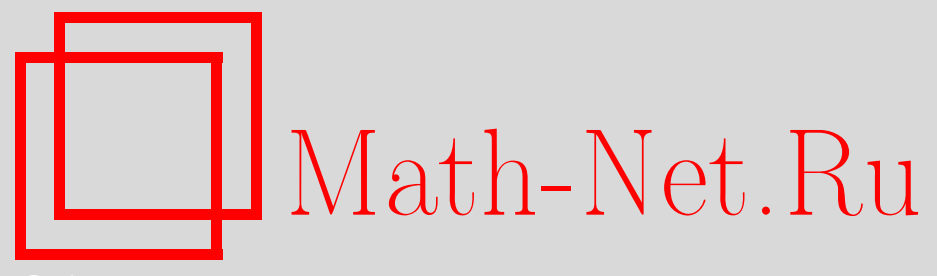

В. А. Иванов, О методе случайного кодирования, Дискрет. матем., 1999, том 11, выпуск 3, 99-108

DOI: https://doi.org/10.4213/dm381

Использование Общероссийского математического портала Math-Net.Ru подразумевает, что вы прочитали и согласны с пользовательским соглашением http://www.mathnet.ru/rus/agreement

Параметры загрузки:

IP : 54.198 .55 .26

26 апреля 2023 г., 10:13:56 
УДК 519.2

\title{
О методе случайного кодирования
}

\author{
(C) 1999 г. . А. Иванов
}

\begin{abstract}
Рассматривается система передачи информации с использованием случайного кодирования сообщений для достижения надежной передачи данных по основному каналу и надежной защиты информации в отводном канале. Получены формулы для вычисления различных вероятностных характеристик таких систем в предположении булевой параметризации поступающих на вход системы сообщений и передаваемых в канал связи сигналов.
\end{abstract}

\section{1. Введение}

В статье А. Вайнера [1], опубликованной в 1975 году, были изучены асимптотические свойства системы передачи информации по каналу связи с отводом. Затем В. И. Коржик и В. А. Яковлев в $[2,3]$ исследовали практические аспекты построения и анализа систем передачи данных при наличии отводного канала утечки информации. В настоящей работе находятся различные вероятностные характеристики таких систем в предположении, что сообщения, поступающие на вход системы, и сигналы, передаваемые в канал связи, представлены в виде векторов, координатами которых являются символы 0 и 1.

Рассмотрим стандартную модель передачи данных по двоичному симметричному каналу. Предположим, что множество

$$
A=\left\{a^{1}, \ldots, a^{M}\right\}
$$

сообщений, поступающих на вход рассматриваемой системы передачи данных, совпадает с множеством $B^{m}$, где $B=(0,1)$, всех двоичных векторов $a^{i}=\left(a^{i 1}, \ldots, a^{i m}\right)$ длины $m, i=0,1, \ldots, M-1, M=2^{m}$, а множество

$$
X=\left\{x^{1}, \ldots, x^{N}\right\}
$$

сигналов, поступающих на вход канала связи, совпадает с множеством $B^{n}$ всех двоичных векторов $x^{j}=\left(x^{j 1}, \ldots, x^{j n}\right) \in B^{n}$ длины $n, n \geqslant m, j=0,1, \ldots, N-1$, $N=2^{n}$.

Пусть на поступающий в канал связи в момент $t$ сигнал

$$
X=\left(x_{t 1}, \ldots, x_{t n}\right) \in B^{n}
$$

воздействует аддитивная случайная помеха

$$
\varkappa_{t}=\left(\varkappa_{t 1}, \ldots, \varkappa_{t n}\right) \in B^{n},
$$


не зависящая от каких бы то ни было сигналов и других помех, координаты которой независимы между собой и распределены по закону

$$
\mathbf{P}\left(\varkappa_{t j}=1\right)=p_{0}=\frac{1-\Delta}{2}, \quad 0 \leqslant \Delta \leqslant 1, \quad j=1, \ldots, n .
$$

Таким образом, на выходе канала с помехами реализуется сигнал

$$
x_{t} \oplus \varkappa_{t}=\left(x_{t 1} \oplus \varkappa_{t 1}, \ldots, x_{t n} \oplus \varkappa_{t n}\right),
$$

по которому требуется восстановить с заданной достоверностью переданное в канал сообщение $a_{t} \in A$.

Для передачи сообщения $a \in A$ обычно используется сигнал $x_{a}$, который определяется с помощью инъективного отображения множества сообщений $A=B^{m}$ в множество сигналов $X=B^{n}$. На приемном конце для восстановления переданного в канал связи сообщения используется подходящее сюзъективное отображение (декодер), задаваемое псевдобулевой функцией $g(x): B^{n} \rightarrow A$, ставящее в соответствие каждому принятому сигналу $x$ вполне определенное сообщение $a=g(x)$.

Рассмотрим теперь ситуацию, когда сообщения передаются одновременно по двум двоично симметричным каналам, основному и отводному (дополнительному, побочному). Предположим, что основной канал абсолютно надежен (то есть $\Delta=1$ и, следовательно, $\left.p_{0}=0\right)$, в то время как отводной канал имеет ограниченную надежность $\left(\Delta \leqslant \Delta_{0}<1,\left(1-\Delta_{0}\right) / 2 \leqslant p_{0} \leqslant 1 / 2\right)$. Требуется построить систему передачи данных, которая бы обеспечивала абсолютно надежную передачу информации по основному каналу и достаточно надежную защиту информации в отводном (побочном) канале.

Далее декодер основного канала будем обозначать символом $f(x)$, а декодер отводного канала символом $g(x)$.

Заметим, что для повышения надежности защиты информации в отводном канале можно применять детерминированное кодирование, максимально размножающее канальные ошибки, неоднородное кодирование, при котором для передачи одного и того же символа $a \in A$ в разные моменты времени выбираются различные сигналы, или случайное кодирование, при котором сигнал, используемый для передачи фиксированного символа $a \in A$, выбирается случайно из заданного множества сигналов. Остановимся более подробно на последнем подходе.

\section{2. Метод случайного кодирования}

Следуя [1], используем для обеспечения надежной защиты информации в отводном канале метод случайного кодирования сообщений. Рассмотрим произвольную псевдобулеву функцию $f(x)$, задающую сюръективное отображение множества сигналов $X$ на множество сообщений $A$. Введем обозначение

$$
X_{a}=X_{a f}=f^{-1}(a)=\{x: f(x)=a\} .
$$

Разбиение

$$
B^{n}=\bigcup_{a} X_{a}
$$

множества всевозможных сигналов $B^{n}$ на непересекающиеся классы назовем кодовой книгой основного канала, а множества $X_{a}=X_{a f}$ страницами кодовой книги. В 
такой интерпретации функция $f(x)$ определяет номер страницы, на которой записан сигнал $x$. Для того чтобы функция $f(x)$ могла быть использована как декодер основного канала, необходимо и достаточно лишь выполнение условия $f\left(x_{a}\right)=a$ для любого сигнала $x_{a}, a \in A$. Воспользуемся этим фактом для построения метода случайного кодирования.

Обозначим $\xi_{a}$ случайный сигнал, равномерно распределенный на странице $X_{a}$ кодовой книги, определяемой функцией $f(x)$. Ясно, что при таком выборе случайной величины $\xi_{a}$, который будем называть равномерным случайным кодированием, при любом $a \in A$ выполняется тождественное соотношение $f\left(\xi_{a}\right)=a$. Поэтому при передаче по основному каналу произвольного сообщения $a$ с помощью случайного сигнала $\xi_{a}$ на выходе декодера $f$ получим с абсолютной достоверностью переданное сообщение $a$. Таким образом, псевдобулева функция $f$ однозначно задает метод равномерного случайного кодирования и является декодером основного канала. Очевидно, что функция $f(x)$ будет также наилучшим декодером для отводного канала передачи в области достаточно малых помех. Однако в общем случае при использовании произвольной функции $g(x)$ для декодирования сигналов, поступающих на выход отводного канала, получаем сообщение $\hat{a}=g\left(\xi_{a} \oplus \varkappa\right)$, которое может оказаться более достоверным, чем сообщение $f\left(\xi_{a} \oplus \varkappa\right)$.

Далее будем полагать, что на вход системы передачи данных поступает последовательность назывисимых равномерно распределенных сообщений $\alpha_{t}$, а функция $f(x)$, задающая случайное кодирование, является равновероятной функцией (каждый прообраз функции $f(x)$ имеет мощность $N / M$ ). Тогда случайная величина $\zeta=\xi_{a}$ при случайном равновероятном выборе сообщения $\alpha$ имеет равномерное распределение, то есть

$$
\mathbf{P}(\zeta=x)=\sum_{a} \mathbf{P}\left(\xi_{a}=x\right)=\mathbf{P}(\alpha=f(x)) \mathbf{P}\left(\xi_{f(x)}=x\right)=\frac{1}{N}
$$

для всех $x \in X_{a}$. Поэтому, предполагая, что на вход системы поступает последовательность равновероятных независимых сообщений $\alpha_{t}, t=1,2, \ldots$, на входе канала будем иметь равновероятную последовательность случайных векторов-сигналов $X_{t}$, $t=1,2, \ldots$

\section{3. Оптимальное декодирование сигналов}

Естественно, что при построении системы надежной передачи данных при небольшом уровне канальных помех (при $\Delta \rightarrow 1$ ) функция $g(x)$ должна удовлетворять соотношению $g\left(x_{a}\right)=a$. На остальных векторах $x \neq x_{a}, a \in A$, значения функции могут быть выбраны произвольно. В общем случае, когда интенсивность помех может принимать произвольные значения, никаких предварительных ограничений на функцию $g(x)$ не накладывается.

Надежность передачи данных будем характеризовать вероятностью правильного приема сообщения

$$
P_{c}=\mathbf{P}(f(X)=g(X \oplus \varkappa)) .
$$

Определение 1. Функцию $g^{f}(x)$ назовем оптимальным декодером отводного канала в системе передачи информации со случайным кодированием, задаваемой функцией $f(x)$, если для любой псевдобулевой функции $g(x)$, переводящей множество $B^{n}$ 
в множество $B^{m}$, выполняется соотношение

$$
\mathbf{P}\left(f(X)=g^{f}(X \oplus \varkappa)\right) \geqslant \mathbf{P}(f(X)=g(X \oplus \varkappa)) .
$$

Лемма 1. Для того чтобы функция $g(x)$ была оптималъным декодером отводного канала, достаточно, чтобы на любом наборе $s \in B^{n}$ она принимала значение $g(s)=$ $a \in B^{m}$, для которого справедливы неравенства

$$
\mathbf{P}(f(s \oplus \varkappa=a) \geqslant \mathbf{P}(f(s \oplus \varkappa=b)
$$

при любом $b \in B^{m}, b \neq a$.

Доказательство. Заметим, что в силу равновероятности $X$ для любых псевдобулевых функций $f$ и $g$ справедливо равенство

$$
\mathbf{P}(f(X)=g(X \oplus \varkappa))=\mathbf{P}(f(X \oplus \varkappa)=g(X)) .
$$

Поэтому в силу неравенств (3) справедливы соотношения

$$
\begin{aligned}
\mathbf{P}(f(X)=g(X \oplus \varkappa)) & =\sum_{s} \mathbf{P}(X=s) \mathbf{P}(f(s \oplus \varkappa)=g(s)) \\
& \geqslant \sum_{s} \mathbf{P}(X=s) \mathbf{P}\left(f(s \oplus \varkappa)=g^{\prime}(s)\right)=\mathbf{P}\left(f(X)=g^{\prime}(X \oplus \varkappa)\right)
\end{aligned}
$$

для любой псевдобулевой функции $g^{\prime}(x)$, откуда следует (2).

Следует заметить, что вид оптимального декодера зависит от параметра канала связи $\Delta$. Проиллюстрируем это на примере.

Пример 1. Пусть бинарный символ $a \in B$ передается по отводному каналу с помощью случайного кода, задаваемого булевой функцией

$$
\begin{aligned}
f(x) & =f\left(x_{1}, x_{2}, x_{3}, x_{4}\right) \\
& =x_{1} x_{2}\left(x_{3} \oplus x_{4} \oplus 1\right) \oplus x_{1} x_{3}\left(x_{4} \oplus 1\right) \oplus x_{2} x_{3} \oplus x_{2} x_{4} \oplus x_{3} x_{4} .
\end{aligned}
$$

В [4] показано, что в различных зонах изменения параметра $\Delta$ оптимальное декодирование достигается на различных функциях. Так, в области изменения вероятности помехи $0 \leqslant p=p_{0}=(1-\Delta) / 2 \leqslant p_{1}$ наилучшее декодирование достигается на функции $g^{f}(x)=f(x)$, а в области $p_{1} \leqslant p \leqslant p_{2}$ наилучшее декодирование достигается на функции $g^{f}(x)=f_{1}(x)$, в области $p_{2} \leqslant p \leqslant 1 / 2$ наилучшее декодирование достигается на функции $g^{f}(x)=f_{2}(x)$, где

$$
\begin{aligned}
p_{1} & \approx 0,259 \\
p_{2} & \approx 0,352 \\
f_{1}(x) & =f(x) x_{1} x_{2} x_{3} x_{4} \\
f_{2}(x) & =x_{2} x_{3} \oplus x_{2} x_{4} \oplus x_{3} x_{4} .
\end{aligned}
$$

Граничные значения $p_{1}$ и $p_{2}$ являются корнями кубических уравнений

$$
2 p^{3}-4 p+1=0, \quad 2 p^{3}-4 p^{2}+4 p+1=0
$$


соответственно. Вероятность правильного декодирования в этих областях выражается формулой

$$
\mathbf{P}\left(g^{f}(x \oplus \varkappa)=f(x)\right)=d_{0}+d_{1} \Delta+d_{2} \Delta^{2}+d_{3} \Delta^{3}+d_{4} \Delta^{4},
$$

где для функции $g^{f}(x)=\psi(x)$ коэффициенты $d(\psi)=\left(d_{0}, d_{1}, d_{2}, d_{3}, d_{4}\right)$ принимают следующие значения:

$$
\begin{aligned}
d(f) & =\frac{1}{16}(8,3 / 2,7 / 2,5 / 2,1 / 2), \\
d\left(f_{1}\right) & =\frac{1}{16}(8,9 / 4,9 / 4,7 / 4,3 / 4) \\
d\left(f_{2}\right) & =\frac{1}{16}(8,3,0,1,0) .
\end{aligned}
$$

Разложим вероятность $P_{c}$ правильного приема сообщения (1) в ряд по параметру $\Delta:$

$$
P_{c}=p^{f g}(\Delta)=\mathbf{P}(f(x)=g(x \oplus \varkappa))=\sum_{k=0}^{n} d_{k}(f, g) \Delta^{k}
$$

Найдем представление для коэффициентов $d_{k}=d_{k}(f, g)$ с помощью преобразований Фурье-Адамара подходящих булевых функций. Пусть $f_{y}(x)$ - булева функция, принимающая значение единица на наборах, на которых псевдобулева функция $f(x)$ принимает значение $y$, и значение нуль на остальных наборах,

$$
\hat{\psi}(u)=\frac{1}{2} \sum_{x} \psi(x)(-1)^{(u, x)}, \quad u, x \in B^{n}
$$

- преобразование Фурье-Адамара булевой функции $\psi(x)$.

Теорема 1. Вероятность правилъного приема сообщения на выходе отводного канала с помощью декодера $g(x)$ в системе передачи со случайным кодированием, задаваемым псевдобулевой функуией $f(x)$, может быть представлена в виде

$$
P_{c}=P^{f g}(\Delta)=\mathbf{P}(f(x)=g(x \oplus \varkappa))=\sum_{k=0}^{n_{k}} d_{k}(f, g) \Delta^{k}
$$

¿de

$$
d_{k}(f, g)=\sum_{y} \sum_{\|u\|=k} \hat{f}_{y}(u) \hat{g}_{y}(u), \quad\|u\|=u_{1}+\ldots+u_{n}
$$


Доказательство. Справедлива цепочка равенств

$$
\begin{aligned}
P^{f g}(\Delta) & =\mathbf{P}(f(X)=g(X \oplus \varkappa))=\sum_{y \in A} \mathbf{P}(f(X)=g(X \oplus \varkappa)=y) \\
& =\sum_{y \in A} \mathbf{P}(f(X)=y, g(X \oplus \varkappa)=y)=\sum_{y \in A} \mathbf{P}\left(f_{y}(X)=1, g_{y}(X \oplus \varkappa)=1\right) \\
& =\sum_{y \in A} \mathbf{P}\left(f_{y}(X) g_{y}(X \oplus \varkappa)=1\right)=\sum_{y \in A} \mathbf{E} f_{y}(X) g_{y}(X \oplus \varkappa) \\
& =\sum_{y \in A} \mathbf{E}\left(\sum_{u \in X} \hat{f}_{y}(u)(-1)^{(u, X)}\right)\left(\sum_{v \in X} \hat{g}_{y}(v)(-1)^{(v, X \oplus \varkappa)}\right) \\
& =\sum_{y \in A} \sum_{u \in X} \hat{f}_{y}(u) \hat{g}_{y}(u) \mathbf{E}(-1)^{(u, \varkappa)} \\
& =\sum_{y \in A} \sum_{k=0}^{n} \sum_{\|u\|=k} \hat{f}_{y}(u) \hat{g}_{y}(u) \Delta^{k} \\
& =\sum_{k=0}^{n} d_{k}(f, g) \Delta^{k},
\end{aligned}
$$

где $\mathbf{E}$ - символ математического ожидания. Таким образом, с учетом равенства

$$
\mathbf{E}(-1)^{(u, \varkappa)}=\Delta^{\|u\|}
$$

получаем (4).

\section{4. Оптимизация метода случайного кодирования}

$\mathrm{C}$ точки зрения обеспечения защиты информации в отводном канале предпочтение следует отдавать методам случайного кодирования, для которых вероятность правильного декодирования переданного сообщения принимает наименьшее значение. С учетом этого введем понятие оптимального случайного кодирования.

Определение 2. Случайное кодирование сообщений, задаваемое функцией $\lambda(x)$, будем называть оптимальным, если для любой псевдобулевой функции $f$, отображающей множество $B^{n}$ в множество $B^{m}$, выполняется неравенство

$$
\mathbf{P}\left(\lambda(X)=g^{\lambda}(X \oplus \varkappa)\right) \leqslant \mathbf{P}\left(f(X)=g^{f}(X \oplus \varkappa)\right) .
$$

Исследуем наиболее простой случай $m=1, n \geqslant 2$.

Теорема 2. Пусть $m=1, n>1$. Тогда наилучшую защиту информации в отводном канале дает метод случайного кодирования, задаваемый булевой функцией $f(x)=x_{1} \oplus \ldots \oplus x_{n}$. В этом случае вероятность правильного приема равна

$$
\mathbf{P}\left(f\left(\xi_{a} \oplus \varkappa\right)=a\right)=\frac{1+\Delta^{n}}{2}
$$


Доказательство. Воспользуемся формулой

$$
\mathbf{P}(f(X) \neq g(X \oplus \varkappa))=\hat{f}(0)+\hat{g}(0)-2 \sum_{k=0}^{n} \alpha^{f, g}(k) \Delta^{k},
$$

где

$$
\alpha^{f, g}(k)=\sum_{\|u\|=k} \hat{f}(u) \hat{g}(u), \quad \hat{f}(u)=\frac{1}{N} \sum_{x} f(x)(-1)^{(u, x)}, \quad N=2^{n} .
$$

Из формулы (6) следует, что разложение (4) по степеням $\Delta$ будет иметь максимальный порядок $\Delta^{n}$ для любой функции $g(x)$, если будут выполняться соотношения

$$
\alpha^{f, g}(k)=0, \quad k=1, \ldots, n-1 .
$$

Для этого достаточно потребовать выполнения равенства $\hat{f}(u)=0$ для всех наборов $u$ веса $k=1, \ldots, n-1$, или, другими словами, потребовать, чтобы функция $f(x)$ допускала представление

$$
f(x)=\hat{f}(0)+(-1)^{\delta(x)} \hat{f}(e),
$$

где $e=(1, \ldots, 1)$ - вектор, состоящий из $n$ единиц, $\delta(x)=(e, x)=x_{1} \oplus \ldots \oplus x_{n}$. Такой функцией будет линейная функция $f(x)=x_{1} \oplus \ldots \oplus x_{n}$, либо функция $f(x)=$ $x_{1} \oplus \ldots \oplus x_{n} \oplus 1$. Заметим, наконец, что из соотношения

$$
f(x \oplus \varkappa) \oplus g(x)=f(x) \oplus g(x) \oplus \varkappa_{1} \oplus \ldots \oplus \varkappa_{n}
$$

следует равенство $g^{f}(x)=f(x)$. Теорема доказана.

Введем следующую общую характеристику метода случайного кодирования.

Определение 3. Назовем коэффициентом защищенности информации для случайного кодирования с декодером основного канала $f$ и декодером отводного канала $g$ натуральное число $r=r(f, g)$, для которого выполняются соотношения

$$
d_{k}(f, g)=0, \quad k=1, \ldots, r-1, \quad d_{r}(f, g)>0 .
$$

Исследуем коэффициент защищенности информации в случае $m>1$ для некоторых конкретных псевдобулевых функций $f(x)$ для случая $f(x)=g(x)$. Как показали вычисления на ЭВМ, оптимальный декодер для рассмотренных примеров с $m=4$, $n=8$ для значений параметра $\Delta=0,2 ; 0,1 ; 0,01$ совпадает с функцией $g^{f}(x)=f(x)$.

Найдем непосредственным вычислением на ЭВМ по формулам (3) и (4) коэффициенты защищенности информации для некоторых конкретных систем передачи сообщений (полубайтов)

$$
a=\left(a_{1}, \ldots, a_{4}\right) \in B^{4}
$$

с помощью сигналов (байтов)

$$
x=\left(x_{1}, \ldots, x_{8}\right) \in B^{8}
$$

при значениях параметра $\Delta=0,2 ; 0,1 ; 0,01$. 
Пример 2. Пусть

$$
f^{1}\left(x_{1}, \ldots, x_{8}\right)=\left(x_{1} \oplus x_{5}, \ldots, x_{4} \oplus x_{8}\right) .
$$

Для случайного кодирования сообщения $a$ применим отображение $x=(\xi, a \oplus \xi)$, где искусственный шум $\xi=\left(\xi_{1}, \ldots, \xi_{4}\right) \in B^{4}$ представляет собой последовательность независимых и равномерно распределенных бинарных случайных векторов. Для указанных значений параметра $\Delta$ получаем $g^{f}=f$ и коэффициенты $d\left(f^{j}\right)=$ $\left(d_{0}\left(f^{j}\right), \ldots, d_{n}\left(f^{j}\right)\right)$ в разложении (3) принимают вид $d\left(f^{1}\right)=\frac{1}{16}(1,0,4,0,6,0,4,0,1)$, вероятность правильного приема $P_{c}=P(\Delta)$ равна

$$
P(0,2)=0,073116, \quad P(0,1)=0,065038, \quad P(0,01)=0,062525,
$$

и $g^{f}=f, r(f, f)=2$.

Пример 3. Рассмотрим код Хэмминга $(7,4,3)$ (см. [5]), сопоставляющий двоичным сообщениям $a=\left(a_{1}, \ldots, a_{4}\right)$, занумерованным числами $0,1, \ldots, 15$, где номер $j(a)$ сообщения $a$ определяется по формуле

$$
j(a)=a_{1}+2 a_{2}+4 a_{3}+8 a_{4},
$$

кодовые слова

$$
x^{j}=\left(x_{1}^{j}, \ldots, x_{7}^{j}\right), \quad j=0,1, \ldots, 15,
$$

вида

$$
\begin{aligned}
x^{0}=0 \rightarrow 0000000, & x^{8}=67 \rightarrow 1000011, \\
x^{1}=15 \rightarrow 0001111, & x^{9}=76 \rightarrow 1001100, \\
x^{2}=22 \rightarrow 0010110, & x^{10}=85 \rightarrow 1010101, \\
x^{3}=25 \rightarrow 0011001, & x^{11}=90 \rightarrow 1011010, \\
x^{4}=37 \rightarrow 0100101, & x^{12}=102 \rightarrow 1100110, \\
x^{5}=42 \rightarrow 0101010, & x^{13}=105 \rightarrow 1101001, \\
x^{6}=51 \rightarrow 0110011, & x^{14}=112 \rightarrow 1110000, \\
x^{7}=60 \rightarrow 0111100, & x^{15}=127 \rightarrow 1111111 .
\end{aligned}
$$

Построим псевдобулеву функцию $f_{H}(x)=f_{H}\left(x_{1}, \ldots, x_{7}\right)$, принимающую значения $y=\left(y_{1}, \ldots, y_{4}\right) \in B^{4}$ или равносильные им значения $j(y)=0,1, \ldots, 15$ на набоpax $x$, для которых выполняется условие $\left\|x \oplus x^{j}\right\| \leqslant 1$, где символом $\|x\|$ обозначается вес Хэмминга $x_{1}+\ldots+x_{7}$ вектора $x$. По функции $f_{H}(x)$ построим псевдобулеву функцию

$$
f^{2}(x)=f_{H}(x) \oplus\left(x_{8}, x_{8}, x_{8}, x_{8}\right)=\left(y \oplus x_{8}, \ldots, y_{4} \oplus x_{8}\right)
$$

от восьми булевых аргументов $x_{1}, \ldots, x_{8}$. Вероятностные характеристики случайного кода, порождаемого псевдобулевой функцией $f^{2}(x)$, принимают значения

$$
\begin{gathered}
d\left(f^{2}\right)=\frac{1}{16}(1 ; 0 ; 9,1875 ; 0 ; 2,1875 ; 0 ; 3,0625 ; 0 ; 0,5625), \\
P(0,2)=0,085700, \quad P(0,1)=0,068256, \quad P(0,01)=0,062557, \\
g^{f}=f, \quad r(f, f)=2 .
\end{gathered}
$$


Пример 4. Пусть

$f^{3}\left(x_{1}, \ldots, x_{8}\right)=\left(x_{2} \oplus x_{3} \oplus x_{4} \oplus x_{5}, x_{3} \oplus x_{4} \oplus x_{1} \oplus x_{6}, x_{4} \oplus x_{1} \oplus x_{2} \oplus x_{7}, x_{1} \oplus x_{2} \oplus x_{3} \oplus x_{8}\right)$.

Для случайного кодирования сообщения $a$ можно применить отображение

$$
x=\left(\xi_{1}, \ldots, \xi_{4}, a_{1} \oplus \xi_{2} \oplus \ldots \oplus \xi_{4}, \ldots, a_{4} \oplus \xi_{1} \oplus \ldots \oplus \xi_{3}\right),
$$

где искусственный шум $\xi=\left(\xi_{1}, \ldots, \xi_{4}\right) \in B^{4}$ представляет собой последовательность независимых и равномерно распределенных бинарных случайных векторов. При указанных значениях параметра $\Delta$ получаем, что $g^{f}=f$ и

$$
\begin{gathered}
d\left(f^{3}\right)=\frac{1}{16}(1,0,0,0,14,0,0,0,1), \\
P(0,2)=0,063900, \quad P(0,1)=0,062587, \quad P(0,01)=0,062500, \\
g^{f}=f, \quad r(f, f)=4 .
\end{gathered}
$$

Заметим, что линейные функции, определяющие координаты псевдобулевой функции $f^{4}(x)=\left(\psi_{1}(x), \ldots, \psi_{4}(x)\right)$, представляют собой скалярные произведения

$$
\psi_{j}(x)=\left(l_{j}, x\right), \quad j=1,2,3,4,
$$

векторов $l_{j}$ базиса кода Хэмминга $(8,4,4)$ (см. [5]) и векторов $x=\left(x_{1}, \ldots, x_{8}\right)$.

\section{5. Системы с линейным декодированием в основном канале}

Запишем функцию $f(x)$ в виде

$$
f(x)=\left(\psi_{1}(x), \ldots, \psi_{m}(x)\right)
$$

Обозначим

$$
l_{j}(x)=\left(l_{j}, x\right)=l_{j 1} x_{1} \oplus \ldots \oplus l_{j n} x_{n}, \quad j=1, \ldots, m,
$$

скалярное произведение двоичного вектора $l_{j}=\left(l_{j 1}, \ldots, l_{j n}\right)$ на двоичный вектор $x=\left(x_{1}, \ldots, x_{n}\right)$, являющееся линейной булевой функцией от $n$ аргументов, $\|x\|=$ $x_{1}+\ldots+x_{n}-$ вес Хэмминга вектора $x$,

$$
d=d\left(l_{1}, \ldots, l_{m}\right)=\min _{c \neq 0}\left\|c_{1} l_{1} \oplus \ldots \oplus c_{m} l_{m}\right\|
$$

где $c=\left(c_{1}, \ldots, c_{m}\right)-$ двоичный вектор длины $m$.

Теорема 3. Пусть для декодирования случайного кода по основному и отводному каналам используется линейная функиия

$$
f(x)=l(x)=\left(l_{1}(x), \ldots, l_{m}(x)\right) .
$$

Тогда коэффищиент защищенности информации равен $r(f, f)=d\left(l_{1}, \ldots, l_{m}\right)$. 
Доказателъство. Представим функцию $f_{y}(x)$ в виде

$$
f_{y}(x)=\frac{1}{2^{m}} \prod_{j=1}^{m}\left(1+(-1)^{y_{j} \oplus \psi_{j}(x)}\right) .
$$

Полагая в $(8) \psi_{j}(x)=\left(l_{j}, x\right)$ и перемножая скобки, получим разложение функции $f_{y}(x)$ в ряд Фурье-Адамара. После перемножения скобок в (8) получим сумму слагаемых вида

$$
(-1)^{y_{j(1)} \oplus \ldots \oplus y_{j(k)} \oplus\left(l_{j(1)}, x\right) \oplus \ldots \oplus\left(l_{j(k)}, x\right)}
$$

для всевозможных наборов натуральных чисел

$$
j(1), j(2), \ldots, j(k), \quad 1 \leqslant j(1)<j(2)<\ldots<j(k) \leqslant m, \quad k=0,1, \ldots, m .
$$

Из того, что для любого двоичного вектора $c=\left(c_{1}, \ldots, c_{m}\right)$ выполняются неравенства

$$
\left\|c_{1} l_{1} \oplus \ldots \oplus c_{m} l_{m}\right\| \geqslant d
$$

где $d=d\left(l_{1}, \ldots, l_{m}\right)$, следует справедливость соотношений $\hat{f}_{y}(u)=0$ для $\|u\| \leqslant d$ и $\hat{f}_{y}(u) \neq 0$ для любого набора $u$ веса $\|u\|=d$, вид которого задается вектором $c$, на котором достигается равенство $\left\|c_{1} l_{1} \oplus \ldots \oplus c_{m} l_{m}\right\|=d$. Теорема доказана.

Следствие 1. Для любого линейного двоичного кода $(n, m, d)$ существует псевдобулева функция $f(x)$, отображающая множество $B^{n}$ в множество $B^{m}$ так, что порождаемое ею случайное кодирование имеет коэффищиент защищенности информачии $r(f, f)=d$.

Таким образом, для построения метода случайного кодирования сообщений $a \in$ $B^{m}$ с помощью сигналов $x \in B^{n}$ с коэффициентом защищенности информации $r(f, f)=d$ достаточно найти код $(n, m, d)$, по векторам его базиса $l_{1}, \ldots, l_{m}$ построить псевдобулеву функцию $f(x)=\left(\left(l_{1}, x\right), \ldots,\left(l_{m}, x\right)\right)$, задающую алгоритм случайного кодирования и проверить оптимальность $g^{f}=f$ декодирования в отводном канале в заданной зоне изменения параметра $\Delta$.

\section{Список литературы}

1. Wyner A., The wire-tap channal. Bell System Technical J. (1975) 54, 1355-1387.

2. Коржик В. И., Яковлев В. А., Пропускная способность канала связи с внутренним случайным кодированием. ІІробл. передачи информации (1992) 28, №4, 24-34.

3. Коржик В. И., Яковлев В. А., Защита информации от утечки за счет побочных электромагнитных излучений и наводок на основе способа кодового зашумления. Информатика и вычислительная техника (1993) 1-2, 61-66.

4. Иванов В. А., Автоматные преобразования случайных последовательностей. Труды по дискретной математике (1998) 2, 151-168.

5. Питерсон У., Уэлдон Э., Кодъ, исправляющие ошибки. Мир, Москва, 1976. 